\title{
On mathematical modelling of insect flight dynamics in the context of micro air vehicles
}

\author{
Rafał Żbikowski 1 †, Salman A. Ansari舟and Kevin Knowles \\ Department of Aerospace, Power and Sensors, \\ Cranfield University \\ Defence Academy of the UK \\ Shrivenham, SN6 8LA, England \\ E-mail: ${ }^{1}$ R.W.Zbikowski@cranfield.ac.uk
}

\begin{abstract}
.
This paper discusses several aspects of mathematical modelling relevant to the flight dynamics of insect flight in the context of insect-like flapping wing micro air vehicles (MAVs). MAVs are defined as flying vehicles ca six inch in size (hand-held) and are developed to reconnoitre in confined spaces (inside buildings, tunnels etc). This requires power-efficient, highly-manoeuvrable, low-speed flight with stable hover. All of these attributes are present in insect flight and hence the focus of reproducing the functionality of insect flight by engineering means. This can only be achieved if qualitative insight is accompanied by appropriate quantitative analysis, especially in the context of flight dynamics, as flight dynamics underpin the desirable manoeuvrability.

We consider two aspects of mathematical modelling for insect flight dynamics.

The first one is theoretical (computational), as opposed to empirical, generation of the aerodynamic data required for the six-degrees-of-freedom equations of motion. For these purposes we first explain insect wing kinematics and the salient features of the corresponding flow. In this context, we show that aerodynamic modelling is a feasible option for certain flight regimes, focussing on a successful example of modelling hover. Such modelling progresses from first principles of fluid mechanics, but relies on simplifications justified by the known flow phenomenology and/or geometric and kinematic symmetries. In particular, this is relevant to six types of fundamental manoeuvres, which we define as those steady flight conditions for which only one component of both the translational and rotational body velocities is non-zero (and constant).

The second aspect of mathematical modelling for insect flight dynamics addressed here deals with the periodic character of the aerodynamic force and moment production. This leads to consideration of the types of solutions of nonlinear equations forced by nonlinear oscillations. In particular, the existence of non-periodic solutions of equations of motion is of practical interest, since this allows steady recitilinear flight.

Progress in both aspects of mathematical modelling for insect flight will require further advances in aerodynamics of insect-like flapping. Improved aerodynamic modelling and computational fluid dynamics (CFD) calculations are required. These theoretical advances must be accompanied by further flow visualisation and measurement to validate both the aerodynamic modelling and CFD predictions.
\end{abstract}

$\dagger$ Principal Research Officer

$\ddagger$ Post-Doctoral Research Officer

$\S$ Head, Aeromechanical Systems Group 


\section{Introduction}

Micro air vehicles (MAVs) are defined as flying vehicles ca six inch in size (hand-held) and are developed to reconnoitre in confined spaces (inside buildings, tunnels etc). This requires power-efficient, highly-manoeuvrable, low-speed flight with stable hover. Such performance is routinely exhibited by flying insects and hence the focus on emulating insectlike flapping by engineering means. A detailed discussion of the future utility of MAVs and the advantages of considering insect-like flapping wing propulsion has been presented elsewhere (Żbikowski 1999a, Żbikowski 1999b). One key aspect is excellent manoeuvrability of insects which calls for examination of their flight dynamics and is the focus of the present paper. Our work on insect-like flapping wing MAVs has advanced the aeromechanics of hover through aerodynamics modelling (Pedersen 2003, Ansari 2004) and also design and manufacture of flapping mechanisms on the MAV scale (Żbikowski et al. 2004, Żbikowski et al. 2005, Galiński \& Żbikowski 2005). While these studies are being extended to flight regimes other than hover, the existing knowledge already allows us to throw some light on the flight dynamics questions involved. The main purpose of this paper is to elucidate the consequent mathematical modelling problems and suggest some approaches to address these problems.

The aim here is to apply insights into insect flight dynamics to MAV design, so the starting point must be investigation of the mechanisms employed by insects to achieve their remarkable performance. Investigating the flight dynamics and control of insects, as opposed to man-made vehicles, poses its own unique problems, limiting applicability of a large body of the relevant aeronautical knowledge. The first and foremost difficulties are experimental in nature, exacerbated by the fact that insects are small and fragile (Taylor \& Thomas 2003). However, the key issue is that neither wind tunnel, nor free-flight testing can be done satisfactorily with the present state-of-the-art, especially from the point of view of meaningful aerodynamic force and moment measurement. In standard aeronautical wind tunnel testing, a model is mounted on a force balance which, in turn, is placed on a moving platform, thus allowing direct force and moment measurement, while controlling the model's orientation with respect to the tunnel's airflow. There are two difficulties with this standard aeronautical set-up in the context of insect flight. Firstly, one cannot make a distinction between the pilot and the airframe-the insect flight control system cannot be switched off to investigate open-loop dynamics. Secondly, insect flight behaviour is influenced by many of its sensory modalities, including vision (Żbikowski 2004), and it is difficult to provide artificial stimulation that faithfully reproduces all forms of natural excitation at the same time. In free-flight testing the second difficulty is mitigated, but at the price of losing the possibility of direct force/moment measurement.

These experimental challenges have counter-intuitive consequences for theoretical analysis. It is not unreasonable to represent the insect flight dynamics by the standard sixdegrees-of-freedom (6dof) equations of motion, so the fundamental question is then: "What do these equations represent?". Since it is impossible to switch off the insect's flight control system, the conventional approach of deriving the open-loop dynamics, then postulating a 
feedback control law and closing the loop is unfeasible. Moreover, unless all of the sensory modalities are artificially stimulated in a manner consistent with natural flight, the insect's control system may be confused. Indeed, the animal will beat its wings as it would normally do in free flight, but some of the expected flight dynamic effects will not be detected by the sensors. Hence, the physiologically intact muscles-wings-sensors loop is broken by the constraints of the experiments and what is observed is neither open- nor closed-loop dynamics, but "broken dynamics" (Taylor \& Żbikowski 2005). A new generation of experiments is likely to overcome this problem and will then provide useful data for semi-empirical models. What we want to discuss here is a mathematical approach in which the required data are effectively computed using aerodynamic modelling, thus producing theoretical models from first principles. Such models would be of use in their own right, especially for MAV performance prediction and design. They would also complement semi-empirical models and benefit from validation by the associated experiments.

The remainder of this paper is organised as follows. Section 2 summarises the basics of insect flight, emphasising its unique features both in the wing kinematics employed (Section 2.1) and occurring aerodynamic phenomena (Section 2.2). Based on these aeromechanical fundamentals, Section 3 discusses how these features could be modelled mathematically within the framework of 6dof equations of motion, pointing out the need for an aerodynamic model, suitable for these purposes. Such a state-of-the-art aerodynamic model for hover is then briefly presented in Section 4, followed by conclusions in Section 5 .

\section{Basics of Insect-like Flapping Flight}

Insect flight is based on specific wing kinematics, summarised in Section 2.1, and these special wing motions produce rather unique aerodynamic phenomena, briefly described in Section 2.2 .

\subsection{Wing Kinematics}

The availability of high-speed photography has enabled basic descriptions of the kinematics of insect wings (Ellington 1984, Ennos 1989b, Dickinson et al. 1999). Insects have either one or two pairs of wings. We restrict ourselves to one pair of wings, inspired by observations of two-winged flies (Diptera), and the description that follows is for such an insect. The overall flapping motion is similar to the sculling motion of the oars on a rowboat, consisting essentially of three component motions: sweeping (fore and aft motion), heaving (up and down motion) and pitching (varying incidence). The motions may not be identical for each wing and are defined by time histories of the angles: $\psi_{l}=\psi_{l}(t), \theta_{l}=\theta_{l}(t), \alpha_{l}=\alpha_{l}(t)$ for the sweeping, heaving and pitching of the left wing, and similarly for the right wing: $\psi_{r}=\psi_{r}(t)$, $\theta_{r}=\theta_{r}(t), \alpha_{r}=\alpha_{r}(t)$. Flapping frequency is typically in the range 5-200 Hz.

The wing motion can be divided broadly into two phases-translational and rotational. The translational phase consists of two halfstrokes - the downstroke and the upstroke (see Figure 11). The downstroke refers to the motion of the wing from its rearmost position to 

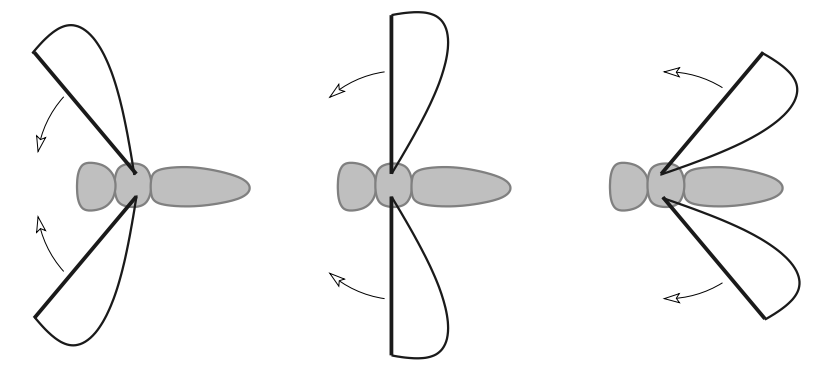

Downstroke
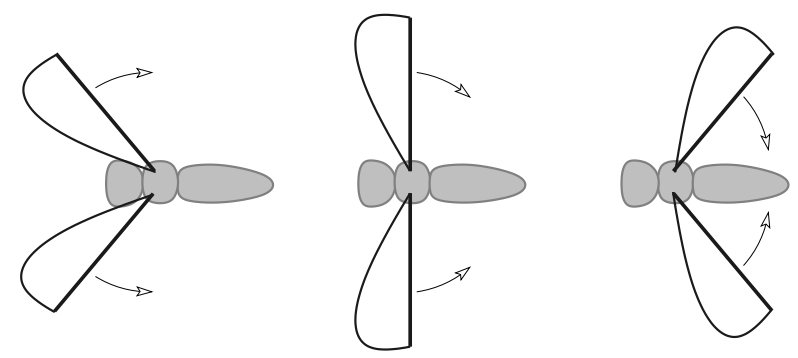

Upstroke

Figure 1. Top view of insect-like flapping

its foremost position, relative to the body. The upstroke describes the return cycle. At either end of the halfstrokes, the rotational phases come into play-stroke reversal occurs, whereby the wing rotates rapidly and reverses direction for the subsequent halfstroke. Strokereversal speeds are typically of the order of hundreds of radians per second (Dickinson 1994) and occupy about 10-20\% of the flapping cycle (Ellington 1984). During this process, the morphological lower surface becomes the upper surface and the leading edge always leads (Figure 1).

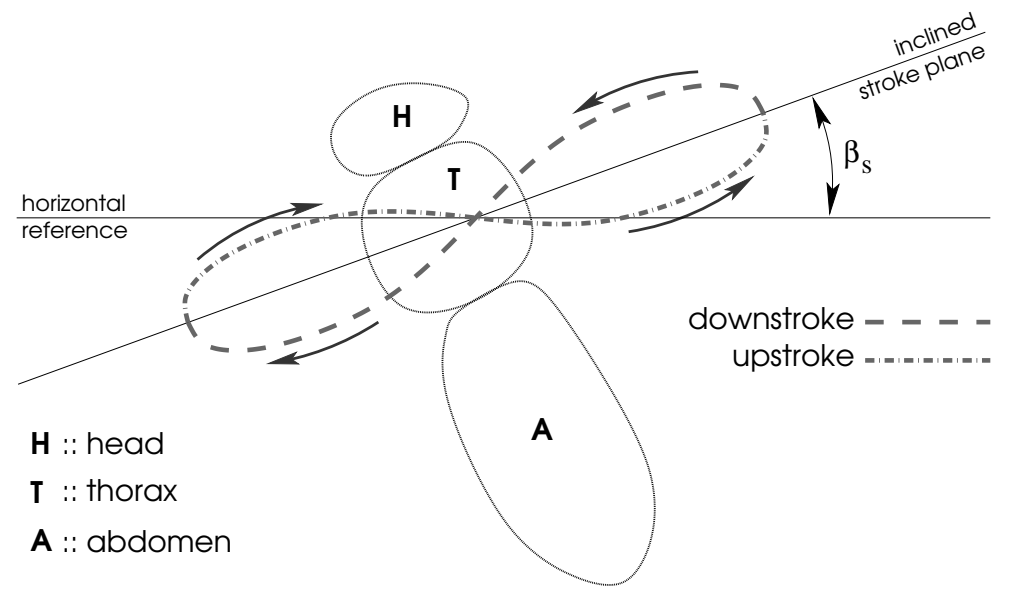

Figure 2. Side view of insect-like flapping

The path traced out by the wing tip (relative to the body) during the wing stroke is similar 
to a figure-of-eight on a spherical surface (see Figure 2) as the wing semi-span is constant. The wing flaps back and forth about a roughly constant plane called the stroke plane (analogous to the tip-path-plane for rotorcraft). The stroke plane is inclined to the horizontal at the strokeplane angle $\beta_{s}$ (Figure 2). The angle swept by the wing during a halfstroke is termed the stroke amplitude.

Since each half-cycle starts from rest and comes to a stop, the temporal evolution of velocity of the flapping wing is non-uniform making the resulting airflow complex. It is also unsteady, i.e. the aerodynamic force varies in amplitude and direction during each wingbeat cycle. The variability of the force is compounded by the influence of the viscosity of air (due to the small scale) and significant interaction of the wing with its own wake, especially in hover.

\subsection{Aerodynamic Phenomena}

The flow associated with insect flapping flight (and scales pertaining to micro air vehicles) is incompressible, laminar, unsteady and occurs at low Reynolds numbers. Despite their short stroke lengths and small Reynolds numbers 1 , insect wings generate forces much higher than their quasi-steady equivalents. Dudley \& Ellington (1990) found that for bumblebees, the quasi-steady estimates of lift and power requirements fail at all flight speeds. In a similar study by Wakeling \& Ellington (1997), the mean lift coefficient required for flight for the dragonfly Sympetrum sanguineum and the damselfly Calopteryx splendens was "reverse-engineered", and in both cases, was found to exceed the maximum possible under quasi-steady conditions.

The motion of a flapping wing differs from conventional aeroplane and rotorcraft wings in that it starts from rest, accelerates to some roughly constant speed and then decelerates to rest again. Stroke reversal occurs and the cycle is repeated. The angle of attack of the wings also changes dramatically during each cycle. The nonlinear nature of this flapping motion and the ability to generate high forces is strongly suggestive of the presence of unsteady aerodynamic phenomena. These are discussed here.

Insect flapping-wing flow is now understood to comprise two components-attached and separated flow (Żbikowski 2002). The attached flow refers to the freestream flow on the aerofoil as well as that due to its unsteady motion (sweeping, heaving and pitching). For insect-like flapping wings, flow-separation is usually observed at both leading and trailing edges - the leading-edge vortex which is bound to the wing for most of the duration of each halfstroke and the trailing-edge wake that leaves smoothly off the trailing edge (see Figure 3). Flow is more or less attached in the remaining regions of the wing.

The leading-edge vortex is now believed to be responsible for the augmented forces observed (Ellington et al. 1996, Liu et al. 1998). It starts close to the wing root and spirals towards the tip where it coalesces with the tip vortex and convects into the trailing wake (Ellington et al. 1996). The overall structure of the leading-edge vortex has been likened to that observed on low-aspect-ratio delta wings (Martin \& Carpenter 1977, van den Berg \&

II Reynolds number $R e$ is defined as $R e=\rho V l / \mu$, where $\rho$ is fluid density, $l$ is a characteristic length (usually wing mean chord), $V$ is a characteristic velocity (usually mean wing-tip speed) and $\mu$ is fluid viscosity. 
Ellington 1997); it is produced and fed by a leading-edge separation.
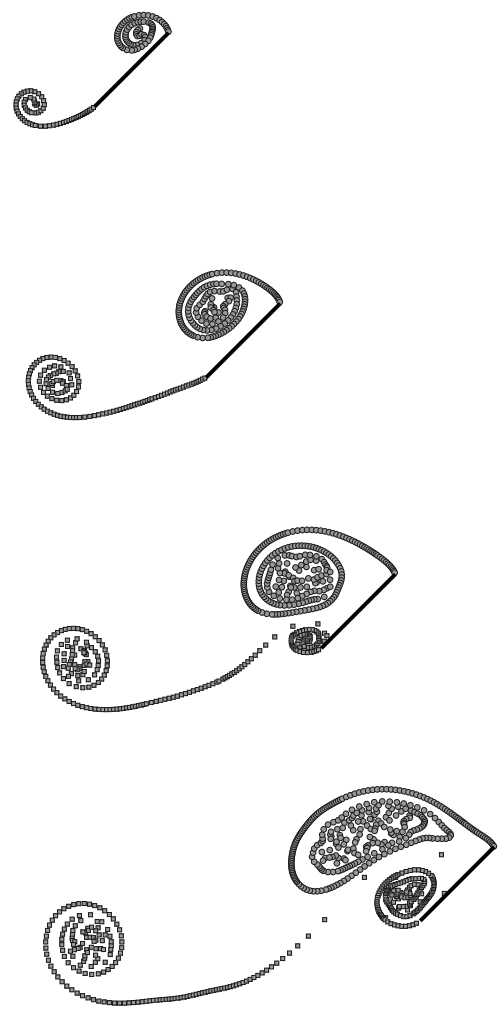
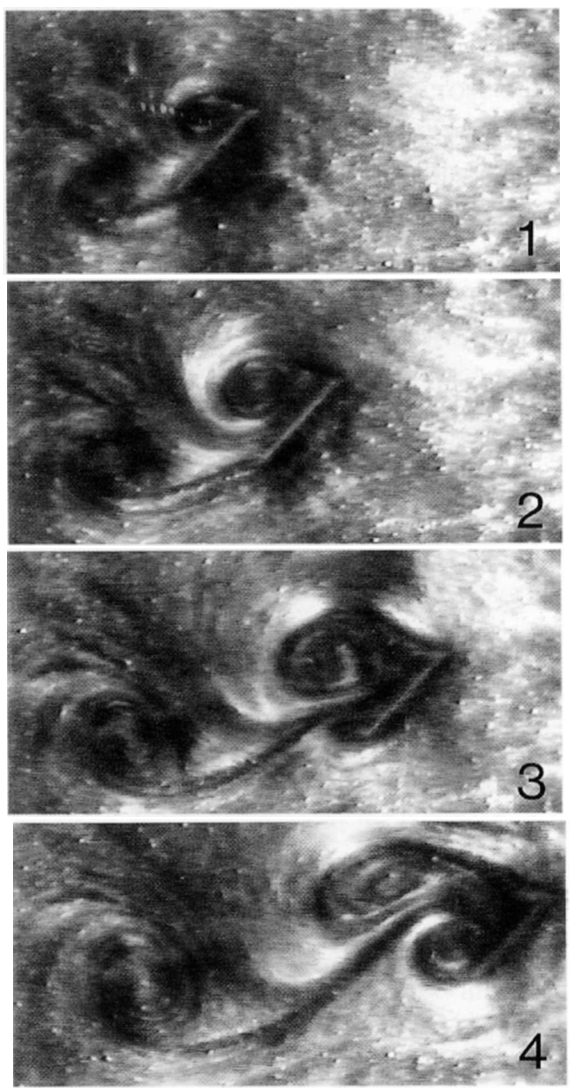

Figure 3. Comparison of flow-visualisation results between theory (Ansari 2004) and experiment (Dickinson \& Götz 1993). The numbers 1 through 4 refer to the number of chord lengths travelled since impulsive start

From the literature, it would be appear that the spanwise spiralling nature of the leadingedge vortex is a more pronounced feature in larger insects (Ellington et al. 1996, Willmott et al. 1997) that operate at higher Reynolds numbers $(R e \sim 5000)$. In their experiments on a dynamically scaled-up model of the much smaller fruit-fly Drosophila melanogaster ( Re 200), Birch \& Dickinson (2001) and Sane \& Dickinson (2001) have also observed a strong leading-edge vortex but with weak spanwise flow.

In hover and slow forward flight, flapping wings are also likely to be affected by the returning wake from previous wingbeats (Dickinson 1994, Dickinson et al. 1999). The importance of this "wake capture" has also been noted by Grodnitsky \& Morozov (1993) who suggested that insects and birds have special mechanisms whereby they extract energy back from their near vortex wake. A similar view was expressed by Ennos (1989a) who speculated that in flies, the kinematics were helped by the aerodynamics.

\section{Flapping flight dynamics modelling}

This section presents mathematical modelling issues for flapping flight within the framework of $6 \mathrm{dof}$ equations of motion. We begin with the basics of flight dynamics in the context of 
insect flight in Section 3.1 and pointing out unique problems arising in the insect context. This is followed by showing, in Section 3.2, the relevance of bifurcation analysis, a state-of-the-art aeronautical technique for manoeuvrable aircraft. By contrast, Section 3.3 focuses on special mathematical tools whose utility for insect flight dynamics is motivated by the periodic nature of aerodynamic force generated by flapping wings.

\subsection{Basics}

Flight dynamics arise because the insect trajectory cannot change instantaneously in response to the aerodynamic force and moment generated by flapping wings. This is due to complex interactions of the inertia of the insect's body with the airflow around the body. Assuming that the body is rigid and longitudinally symmetric, the interactions are quantified by the equations of motion (Roskam 1995, Etkin \& Reid 1996):

$$
\begin{array}{ll}
m(\dot{u}-v r+w q) & =m g_{x}+X \\
m(\dot{v}+u r-w p) & =m g_{y}+Y \\
m(\dot{w}-u q+v p) & =m g_{z}+Z \\
I_{x x} \dot{p}+\left(I_{z z}-I_{y y}\right) q r-I_{x z}(\dot{r}+p q) & =L \\
I_{y y} \dot{q}-\left(I_{z z}-I_{x x}\right) p r+I_{x z}\left(p^{2}-r^{2}\right) & =M \\
I_{z z} \dot{r}+\left(I_{y y}-I_{x x}\right) p q-I_{x z}(\dot{p}-q r) & =N .
\end{array}
$$

For an insect of mass $m$ and inertia tensor $\boldsymbol{I}$ the coordinate system is fixed to its body at the centre of gravity, see Figure 4. The translational velocity is $\boldsymbol{V}=(u, v, w)$ and the angular velocity is $\boldsymbol{\omega}=(p, q, r)$. Also, the aerodynamic force is given by $\boldsymbol{F}=(X, Y, Z)$, the aerodynamic moment by $\boldsymbol{M}=(L, M, N)$, and the gravity vector is resolved as $\boldsymbol{g}=$ $\left(g_{x}, g_{y}, g_{z}\right)$. Equations $(1)-(6)$ can be rewritten in terms of the sideslip angle $\beta=\sin ^{-1}(v / V)$ and the angle of attack $\alpha=\sin ^{-1}(u / V \cos \beta)$, where $V=\sqrt{u^{2}+v^{2}+w^{2}}$ is the speed. Note that $\alpha$ and $\beta$ here refer to the angles between the body axes and the body velocity vector $V$. They should be distinguished from the angles defining the wingbeat kinematics, especially the pitching angles $\alpha_{l}$ and $\alpha_{r}$, and also from the stroke plane angle $\beta_{s}$.

It is convenient to have a short-hand for (1)- (6) in the form:

$$
\dot{\boldsymbol{x}}=f_{o}(\boldsymbol{x}, \boldsymbol{u})
$$

with the state $\boldsymbol{x}=(u, v, w, p, q, r)$ and the control input $\boldsymbol{u}=(X, Y, Z, L, M, N)$. The key to successful flight is purposeful generation of the control input $\boldsymbol{u}$, i.e. aerodynamic force $\boldsymbol{F}$ and moment $\boldsymbol{M}$. The insect controls their production by modulating the flapping of its wings, comparing the actual and required trajectory, so that a feedback loop is formed to follow the required trajectory. Since in equation (7) the control $\boldsymbol{u}$ is not specified as a function of $\boldsymbol{x}$, i.e. the feedback loop has not been defined, it is called open-loop dynamics.

For piloted aircraft, the required trajectory $\boldsymbol{r}$ is known from the aircraft mission and/or flight manual. Similarly, the feedback law $\boldsymbol{\phi}$ is also known, as it was specified when the aircraft was designed. In a manoeuvre, aircraft flight dynamics arise from substituting 


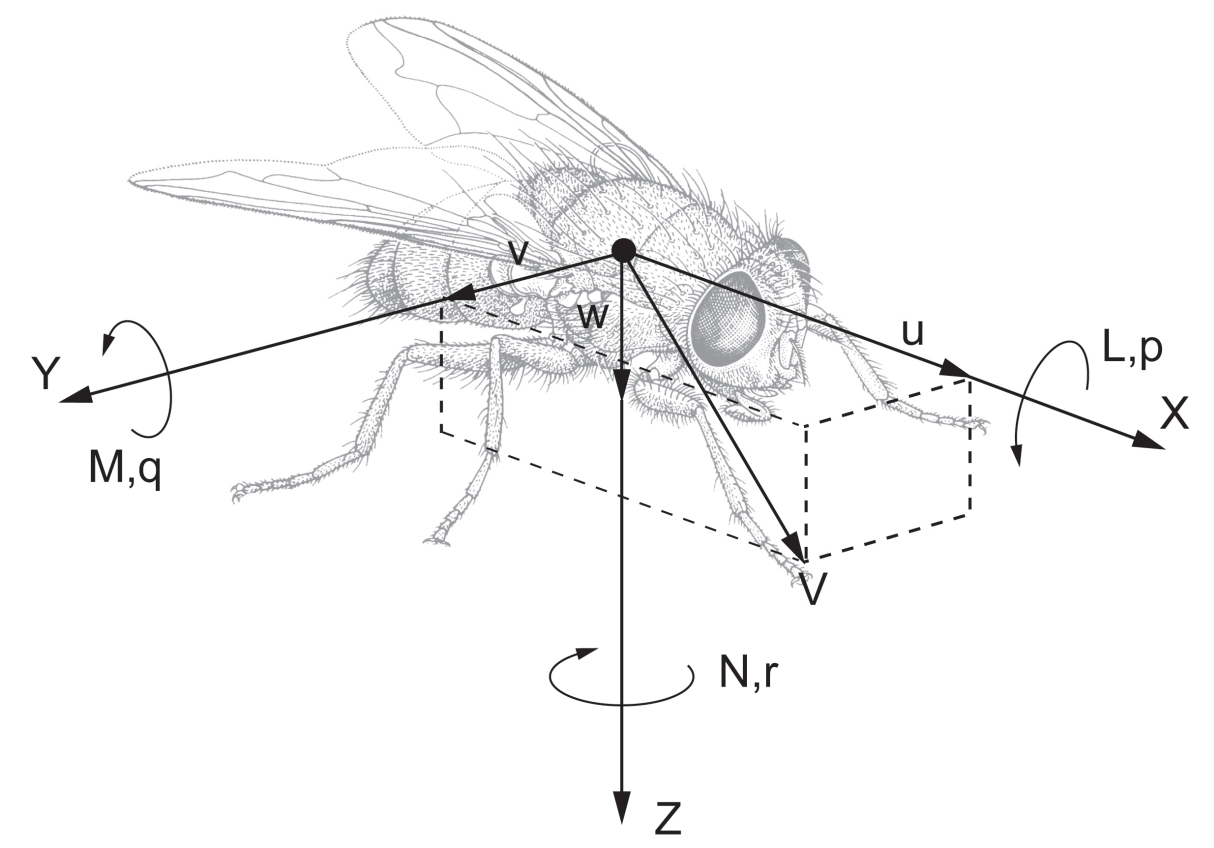

Figure 4. Coordinate system and notation for insect flight dynamics equations.

$\boldsymbol{u}(t)=\boldsymbol{\phi}(\boldsymbol{r}(t), \boldsymbol{x}(t), t)$ in (7) to obtain the closed-loop dynamics:

$$
\dot{\boldsymbol{x}}=\left.\boldsymbol{f}_{o}(\boldsymbol{x}, \boldsymbol{u})\right|_{\boldsymbol{u}=\phi(\boldsymbol{r}, \boldsymbol{x}, t)}=\boldsymbol{f}_{c}(\boldsymbol{x}, t) .
$$

The distinction between the "pilot" and the "aircraft" cannot be made for an insect, and the mission (or "flight manual") can only be speculated about. Hence, the open-loop description (7) cannot be obtained experimentally. However, it may still be possible to deduce the closed-loop description $f_{c}$ to gain conceptual, functional and physical insights into flight dynamics of free-flying, manoeuvrable insects. The types of experiments which are possible and/or desirable are discussed in (Taylor \& Żbikowski 2005). Here, we want to discuss an approach which, to some extent, could be an alternative to the challenging experiments required for investigating insect flight dynamics.

The key difficulty in flight dynamics modelling is finding a correct and tractable relationship between the aerodynamic force $\boldsymbol{F}$ and moment $\boldsymbol{M}$ and their effects on the body kinematics $\boldsymbol{x}$. In the flapping context, $\boldsymbol{F}$ and $\boldsymbol{M}$ are produced by the wingbeat kinematics which, in turn, depend on the body kinematics. Since the insect body lift does not seem to contribute much, $\boldsymbol{F}$ and $\boldsymbol{M}$ are largely produced by the airflow around the flapping wings, resulting from the combined effects of the wingbeat and body kinematics.

In hover, $\boldsymbol{V}=\mathbf{0}$ and $\boldsymbol{\omega}=\mathbf{0}$, so that the coupling between the wingbeat and body kinematics is absent. Then it is possible to derive, from first principles of aerodynamics, how the force and moment are produced. In other words, it is possible to find $\boldsymbol{F}$ and $\boldsymbol{M}$ as functions of wingbeat kinematics under the assumption of wingbeat symmetry: $\psi_{l}=\psi_{r} \triangleq \psi_{\text {hover }}$, $\theta_{l}=\theta_{r} \triangleq \theta_{\text {hover }}, \alpha_{l}=\alpha_{r} \triangleq \alpha_{\text {hover }}$, i.e. effectively to obtain $\boldsymbol{F}=\boldsymbol{F}\left(\psi_{\text {hover }}, \theta_{\text {hover }}, \alpha_{\text {hover }}\right)$ and $\boldsymbol{M}=\boldsymbol{M}\left(\psi_{\text {hover }}, \theta_{\text {hover }}, \alpha_{\text {hover }}\right)$. A summary of such an aerodynamic model is given in Section 4 
For flight regimes other than hover, i.e. when $\boldsymbol{V} \neq \mathbf{0}$ or $\boldsymbol{\omega} \neq \mathbf{0}$, the situation is more complex. For example, steady forward flight is defined by $\boldsymbol{V}=(u, 0,0)$, with $u=$ const, and $\boldsymbol{\omega}=\mathbf{0}$. While the wingbeat kinematics will be symmetric $\psi_{l}=\psi_{r} \triangleq \psi_{f f}, \theta_{l}=\theta_{r} \triangleq \theta_{f f}$, $\alpha_{l}=\alpha_{r} \triangleq \alpha_{f f}$, the force and moment will now be affected by the presence of incident flow on the flapping wings, so that $\boldsymbol{F}=\boldsymbol{F}\left(\psi_{f f}, \theta_{f f}, \alpha_{f f} ; u\right)$ and $\boldsymbol{M}=\boldsymbol{M}\left(\psi_{f f}, \theta_{f f}, \alpha_{f f} ; u\right)$. This situation is more involved than hover, but still results in a steady flight condition, because $\dot{\boldsymbol{V}}=\mathbf{0}$ and $\dot{\boldsymbol{\omega}}=\mathbf{0}$. Also, only one component of both the translational and rotational body velocities affects the flow generated by flapping wings, so that the resulting aerodynamic model will be a limited extension of hover.

These observations about steady forward flight suggest that, within the 6dof framework, there are certain flight modes for which aerodynamic modelling of flapping wings can be obtained by a limited extension of the modelling of hover. We call these flight modes fundamental manoeuvres which are defined as steady flight conditions, i.e. $\dot{\boldsymbol{V}}=\mathbf{0}$ and $\dot{\boldsymbol{\omega}}=\mathbf{0}$, where only one component of both the translational and rotational body velocities is non-zero (and constant). There are six types of fundamental manoeuvres:

(i) three pure translations:

(a) $\boldsymbol{V}=(u, 0,0)$, with $u=$ const, and $\boldsymbol{\omega}=\mathbf{0}$

(b) $\boldsymbol{V}=(0, v, 0)$, with $v=$ const, and $\boldsymbol{\omega}=\mathbf{0}$

(c) $\boldsymbol{V}=(0,0, w)$, with $w=$ const, and $\boldsymbol{\omega}=\mathbf{0}$

(ii) three pure rotations:

(a) $\boldsymbol{\omega}=(p, 0,0)$, with $p=$ const, and $\boldsymbol{V}=\mathbf{0}$

(b) $\boldsymbol{\omega}=(0, q, 0)$, with $q=$ const, and $\boldsymbol{V}=\mathbf{0}$

(c) $\boldsymbol{\omega}=(0,0, r)$, with $r=$ const, and $\boldsymbol{V}=\mathbf{0}$

Their theoretical significance stems not only from the simplification of aerodynamic modelling, but also from the reduced form of the equations of motion (1)-(6). Finally, it is interesting to note that some species of Diptera seem to perform most of their manoeuvres in a stereotyped way by flying trajectories composed from fragments of pure translation followed by pure rotation.

Manoeuvres which entail more than one non-zero component of both the translational and rotational body velocities and/or non-zero accelerations have more complex effects on the flow generated by flapping wings. The resulting interdependence of body and wingbeat kinematics is not likely to be amenable to aerodynamic modelling methods of the type outlined in Section 4. However, it is possible, in principle, to calculate the resulting $\boldsymbol{F}$ and $\boldsymbol{M}$ at a finite number of points on the flight envelope using computational fluid dynamics (CFD) and interpolate between the points.

Thus, either by aerodynamic modelling, or by CFD one can obtain theoretically a representation of $\boldsymbol{F}$ and $\boldsymbol{M}$ as functions of body and wingbeat kinematics over a reasonable flight envelope. We now proceed to explain how such information can be used to analyse the resulting flight dynamics. 


\subsection{Bifurcation analysis}

When the aerodynamic force and moment are balanced by the insect's weight, the insect will be in steady flight. Linearisation of equations (1)-(6) around the steady flight trajectory $\overline{\boldsymbol{x}}$ approximates $(8)$ with

$$
\delta \dot{\boldsymbol{x}}=\boldsymbol{A}_{c} \boldsymbol{\delta} \boldsymbol{x}
$$

where the variable $\delta \boldsymbol{x}=\boldsymbol{x}-\overline{\boldsymbol{x}}$ is a perturbation of the trajectory $\overline{\boldsymbol{x}}$. For a constant trajectory, $\overline{\boldsymbol{x}}(t) \equiv$ const, $\boldsymbol{A}_{c}$ is a constant matrix. If the steady flight is periodic, $\overline{\boldsymbol{x}}(t+T)=\overline{\boldsymbol{x}}(t)$, then the matrix is periodic (time-varying), $\boldsymbol{A}_{c}(t+T)=\boldsymbol{A}_{c}(t)$. Both cases are mathematically tractable (Yakubovich \& Starzhinskii 1975).

In contrast to steady flight, manoeuvres entail the nonlinear, dynamic phenomena of: (i) inertial coupling (Etkin \& Reid 1996), see equations (4)-(6), (ii) aerodynamic cross-coupling (Orlik-Rückemann 1983), i.e. interdependence among the components of $\boldsymbol{F}$ and $\boldsymbol{M}$, and (iii) unsteady aerodynamics (Hancock 1995). Linearisation (9) simplifies away (i) and (ii), but the effects of (iii) can be included, albeit in an approximate fashion, as is now explained using the example of the pitching moment $M$ for longitudinal dynamics (sideslip angle $\beta=0$ ).

The pitching moment $M$ in (9) is assumed to depend linearly on the current value of the angle of attack $\alpha$, i.e. $M(t)=M_{\alpha} \alpha(t)$, where $M_{\alpha}$ is a constant. In manoeuvre, the pitching moment will not change instantaneously in response to changes in the angle of attack, due to the effect of the wake. This dependence of time history (Tobak \& Schiff 1978) is the essence of unsteady aerodynamics and in this example is related to the Wagner effect (Wagner 1925), relevant to insect flight (Żbikowski 2002). The coefficient $M_{\alpha}$ will not be a constant, but a function of time according to the linear integral equation:

$$
M_{\alpha}(t)=M_{\alpha}^{\infty}\left(K(t) \alpha\left(t_{0}\right)+\int_{t_{0}}^{t} K(t-\tau) \alpha^{\prime}(\tau) d \tau\right),
$$

where $M_{\alpha}^{\infty}$ is the steady value. Assuming that the kernel $K$ is known, substitution of (10) into (9) will result in an integro-differential equation and the analysis becomes involved. However, only the recent time history has significant influence, so it is feasible (Tobak et al. 1984, Thomas 1984) to approximate (10) as

$$
M_{\alpha}(t) \approx M_{\alpha} \alpha(t)+M_{\dot{\alpha}} \dot{\alpha}(t),
$$

where both coefficients $M_{\alpha}$ and $M_{\dot{\alpha}}$ are constant.

Inclusion of approximate unsteady effects, as in (11), leads to representing $X, Y, Z$ in (1)-(3) and $L, M, N$ in (4)-(6) as linear combinations of $\delta \boldsymbol{x}$ and $\delta \dot{\boldsymbol{x}}$, so that (9) becomes

$$
\boldsymbol{E} \boldsymbol{\delta} \dot{\boldsymbol{x}}=\boldsymbol{A}_{c} \boldsymbol{\delta} \boldsymbol{x} .
$$

Here the matrix $\boldsymbol{E}$ has constant entries and is invertible, so that both sides of (12) can be multiplied by $\boldsymbol{E}^{-1}$ and the result is mathematically no more involved than 9 ).

Formulae (10) and (11) are valid for small values of $\alpha$. In severe manoeuvres (McCune et al. 1990), the integral equation corresponding to (10) is nonlinear, so that $M_{\alpha}$ and $M_{\dot{\alpha}}$ in (11) depend on $\alpha$ and $\dot{\alpha}$,

$$
M_{\alpha}=M_{\alpha}(\alpha, \dot{\alpha})
$$




$$
M_{\dot{\alpha}}=M_{\dot{\alpha}}(\alpha, \dot{\alpha}) \text {, }
$$

rather than being constant. Such relationships can be established empirically, but in the insect case the associated experiments are more involved than in a conventional set-up. As explained before, this not only due to the smallness and fragility of insects, but—above all—due to the difficulties of accounting for active flight control applied by the insect during the experiment.

The main thrust of this paper is the proposition that an attractive alternative to the experiment is to derive relationships of type (13) theoretically either by aerodynamic modelling or computational fluid dynamics (CFD). If this can be done from first principles, the experimental difficulties of obtaining the aerodynamic data of type (13), required for flight dynamics equations of motion (1)-(6), are avoided.

If relationships of type (13) are available, either from experiment or from theory, a useful approach is to combine the fully nonlinear description (8) with an exhaustive collection of its linear approximations (12), so that both nonlinear and unsteady aspects are captured in a mathematically tractable way. This alternative is realised by bifurcation analysis, a stateof-the-art approach to nonlinear aircraft dynamics (Goman et al. 1997). The validity and usefulness of this approach has been extensively verified over the last twenty years on real data from manoeuvring fighter aircraft: the F-8 (Liaw \& Song 2001, Liaw et al. 2003), the F4 (Carroll \& Mehra 1982, Lowenberg \& Champneys 1998), the F-14 (Jahnke \& Culick 1994), the F-16 (Avanzini \& de Matteis 1997) and the research configurations of their modern successors: the F-18/HARV (Gránásy \& Thomasson 1998, Sinha 2002), HIRM (Patel \& Littleboy 1998, Lowenberg \& Patel 2000).

The first step in bifurcation analysis (Carroll \& Mehra 1982, Jahnke \& Culick 1994) is to calculate all the steady states of the system by setting $\boldsymbol{f}_{c}(\boldsymbol{x}, t) \equiv 0$ in (8). Then, local stability of each steady state is investigated by examining the eigenvalues of the matrix $\boldsymbol{E}^{-1} \boldsymbol{A}_{c}$ in $(12)$ : negative eigenvalues mean that the steady state is stable, positive that it is unstable. Transition from a stable to unstable steady state (or vice versa) means that some eigenvalues will continuously pass through zero. Changes in the stability of a steady state are manifestations of nonlinearity and lead to qualitative changes of dynamic behaviour which are called bifurcations. Such qualitative changes can be analysed by the well-developed mathematics of bifurcation theory (Chow \& Hale 1982, Arnol'd et al. 1999, Demazure 2000), together with the associated numerical and software tools (Allgower \& Georg 1990, Govaerts 2000).

The essence of the proposed modelling approach to insect flight dynamics is: (i) to obtain theoretically a nonlinear, unsteady model (8) with dynamic stability derivatives (13), and (ii) apply bifurcation analysis to the resulting model.

\subsection{Specialised mathematical tools}

In Taylor \& Żbikowski (2005), a semi-empirical nonlinear time-periodic (NLTP) model of the longitudinal flight dynamics of the desert locust was derived. It was based on reduction of equations (1)-(6) to the $x z$-plane while noting that both the aerodynamic force and moment components are periodic. Mathematically, this can be interpreted as a nonlinear system forced by nonlinear oscillations and raises a few questions of theoretical and practical importance. 
Some of these are considered in the discussion section of Taylor \& Żbikowski (2005), especially §§6.5-6.6 (pages 218-220), where conjectures about (i) limit cycle control, (ii) orbital stability and (iii) asymptotically autonomous systems are proposed. Below we consider some new aspects of (i), see Section 3.3.1, and also point out some tools relevant to (iii), see Section 3.3 .2

3.3.1. Synchronisation The NLTP model (Taylor \& Żbikowski 2005) is novel, especially in the context of insect flight dynamics, but at the same time belongs to the large subject of Nonlinear Oscillations. Interestingly, there are at least three issues here that are seldom considered together in the Theory of Oscillations, namely:

(i) when will the solutions be oscillatory?

(ii) when will the solutions not be oscillatory?

(iii) how can (ii) and/or (iii) be effected by a limit cycle control scheme?

There are many mathematical results which throw light on each of these questions separately, but it does not seem immediately obvious how the disparate mathematical techniques involved could be used in an integrated way. An interesting alternative is to go back to the physical and engineering origins of nonlinear oscillations where an integrated approach was a necessity. This alternative goes under the name of "synchronisation", e.g. see (Pikovsky et al. 2001, Fradkov \& Pogromsky 1998, Huijberts \& Nijmeijer 2001). There has been a resurgence of interest in the subject due to the recent interest in chaos theory, but this aspect is not relevant to insect flight dynamics.

Systems as diverse as coupled clocks, flashing fireflies, cardiac pacemakers, firing neurons, and applauding audiences exhibit a tendency to operate in synchrony, i.e. by tuning and retuning their nonlinear oscillations. A precise definition of synchronisation in general is usually either too restrictive or too broad, but includes phase locking and frequency entrainment, periodic forcing or interaction of periodic oscillators and noise-influenced synchronisation. In all cases, the dynamical system splits into subsystems that affect each other by interaction, and the problem is to understand how the interaction determines the dynamics of the system as a whole. In the context of the NLTP model of insect flight dynamics, the phenomena of interest are:

(i) synchronisation of a periodic oscillator by external force

(ii) suppression of oscillations

As for (i)), this entails both phase and frequency locking and can be achieved by applying a weak force, i.e. a small amplitude oscillation. In particular, entrainment can be effected by a weak pulse train. This seems a plausible scheme for the direct muscles actuating insect wings for flight control. Indeed, it is compatible with the limit cycle control hypothesis and also consistent with the asymptotically autonomous character of the control proposed by Taylor \& Żbikowski (2005). This can be explained as follows. The wing-thorax system of the insect oscillates with frequency $\omega_{0}$ and the wings are controlled by direct muscles which are much smaller (weaker) than the thoracic muscles. If the direct muscles act on the wings with a 
weak, periodic force (amplitude $\epsilon$ and frequency $\omega$ ), they can retune the wing-thorax system to a new oscillating frequency wing-thorax $\Omega$. In general, this new frequency is different from the old one and the forcing one as well, $\Omega \neq \omega_{0}$ and $\Omega \neq \omega$. The larger the detuning $\Delta \omega=\omega_{0}-\omega$, the larger the amplitude $\epsilon$ must be to achieve this new $\Omega$. This can be captured precisely and illustrated graphically by the entrainment region in the $\omega-\epsilon$ plane through the so-called Arnol'd tongues (triangle-like regions with vertex at $\omega=\omega_{0}$, widening upwards for increasing values of $\epsilon$ ). Also worth noting, is that this theory is still applicable under random fluctuations, when frequency diffusion is observed, a phenomenon consistent with a range of flapping frequencies (described in $\$ 4.2$ of Taylor \& Żbikowski 2005).

As for (ii), a weak impulse train can be used to suppress oscillations. This means forcing the system off a limit cycle to a neighbouring point equilibrium, in effect a controlled Hopf bifurcation. Indeed, in both synchronisation and suppression, the Poincaré map (stroboscopic observation of nonlinear oscillations) of bifurcation analysis is a tool of choice.

Finally, it is worth mentioning that synchronisation issues are related to the observer theory of nonlinear control (Huijberts \& Nijmeijer 2001). The link is through the Reconstruction Theorem of bifurcation analysis (Sauer et al. 1991) which is a result formalising reconstruction of attractors from data, hence providing a link between nonlinear theory and practical experiment.

3.3.2. Limiting equations It was argued in $\$ 6.4$ of (Taylor \& Żbikowski 2005) that, while the NLTP model is explicitly time-varying, it must-in effect-be asymptotically autonomous (time-invariant). As mentioned in the paper, the idea goes back to Markus (Markus 1956), but there has been important progress in this area (Strauss \& Yorke 1967, Wu 1995). In particular, stability theory has been worked on with some success, e.g. see the appendix of (LaSalle 1976). More broadly, the method of limiting equations has emerged as a technique, rather than an idea (Kato et al. 1996).

The main idea can be illustrated beginning with a non-autonomous ordinary differential equation

$$
\dot{\boldsymbol{x}}=\boldsymbol{f}(t, \boldsymbol{x}), \quad t \geq t_{0},
$$

where the right-hand side is defined for $\boldsymbol{x}$ in some open subset of $\mathbb{R}^{n}$. Suppose that there is a sequence $t_{k} \rightarrow \infty$ such that $\boldsymbol{f}\left(t+t_{k}, \boldsymbol{x}\right) \rightarrow \boldsymbol{g}(t, \boldsymbol{x})$ in the compact-open topology. Then

$$
\dot{\boldsymbol{x}}=\boldsymbol{g}(t, \boldsymbol{x})
$$

is a limiting system of (14). Various properties of the solutions of (14), e.g., boundedness and stability, can be deduced if the class of limiting systems (15) satisfies appropriate conditions. Of particular interest is the case when the limiting equation is itself autonomous, i.e. the righthand side $g$ in (15) does not explicitly depend on time $t, g=g(x)$. For example,

$$
\begin{aligned}
& \dot{x}_{1}=x_{2} \\
& \dot{x}_{2}=x_{1}+x_{2}+x_{1} \sin \sqrt{t}
\end{aligned}
$$

has as its limiting equation

$$
\dot{x}_{1}=x_{2}
$$




$$
\dot{x}_{2}=\mu x_{1}+x_{2},
$$

where $\mu \in[0,2]$. More interestingly,

$$
\dot{\boldsymbol{x}}=\boldsymbol{g}(\boldsymbol{x})+\boldsymbol{h}(t, \boldsymbol{x})
$$

is asymptotically autonomous if for every sequence $\boldsymbol{u}_{k}$ of functions $\boldsymbol{u}_{k}:[a, b] \rightarrow \mathbb{R}^{n}$, continuous on $[a, b]$ and converging to $\boldsymbol{u}_{0}$,

$$
\int_{a}^{b} \boldsymbol{h}\left(t_{k}+s, \boldsymbol{u}_{k}(s)\right) d s \rightarrow 0 .
$$

There are two kinds of results on stability. The first kind deduces stability properties of (14) from stability properties of (15) by constructing a Lyapunov function for (15). The second kind uses a scalar comparison equation for (14). It is assumed that the comparison equation admits a Lyapunov function. A main point is that a Lyapunov-type function is only assumed to exist for the comparison equation; on the other hand, the limiting comparison systems are assumed to have non-positive right-hand sides.

Finally, there are a few (but not many) results pertaining to the feedback control problem. Here the issue is whether a feedback control law designed for (15) will also work for (14). In general, this is a difficult question, but some results on stabilising control can be derived.

\section{Nonlinear, unsteady aerodynamic model of hover}

By virtue of the complex kinematics (see \$2.1), the wake of a flapping wing is nonlinear and cannot be represented adequately by Wagner's (1925) linear kernel in Equation 10. Whereas in conventional aircraft control the wake function may be truncated to include only the recent past (Equation 11), such an assumption is not tenable in insect flight since a significant amount of shed wake is still in the vicinity of the wing, especially during hover. A more advanced model that obviates this truncation while also capturing the nonlinearity of the problem is, therefore, required.

With a view to addressing this problem, Ansari et al. (2006a) developed an analytical, unsteady aerodynamic model that captures the most important aerodynamic phenomena pertaining to insect flapping flight (see $\$ 2.2$ ) and, in doing so, accounts for the nonlinearity in the problem (for full details, see Ansari 2004). The model is circulation-based (Żbikowski 2002) and develops broadly on the seminal work of von Kármán \& Sears (1938), with refinements along the lines of McCune et al. (1990) and McCune \& Tavares (1993), albeit introduced with several significant extensions.

\subsection{Modelling methodology}

The model is quasi-three-dimensional—strip theory is used to divide the wing spanwise into chordwise sections that are treated essentially as two-dimensional. Division of the wing into sections requires closer attention. As also noted by Ansari et al. (2003), the low aspect ratio and high solidity $\| \mathrm{pf}$ insect wings requires that radial chords be used instead of normal

\| Solidity refers to the ratio of wing planform area to area swept by the wing. 
(straight) chords (see Figure 5). This is necessary because each wing section would otherwise 'see' a curved (and significant) incidence velocity. A consequence of this is that each wing section resides in a radial cross-plane which is then unwrapped flat and the flow is solved as a planar two-dimensional problem. The overall contribution of the wing is obtained by integrating along the span.
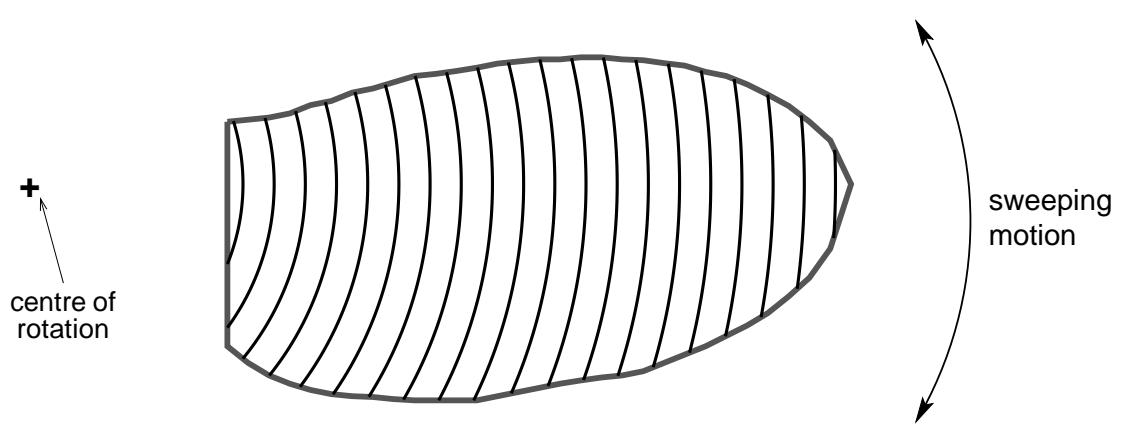

Figure 5. Radial chord representation of a fruit-fly wing

In each 2-D section, the aerofoil is represented by a continuous distribution of bound vorticity and the zero-through-flow condition is enforced on its surface. As noted in $\$ 2.2$ above, insect flight kinematics cause separation from both leading and trailing edges so that the flow may be represented as a combination of attached and separated flows. In the aerodynamic model, therefore, two wakes are shed in the form of free vortex sheets-one each from the leading and trailing edges-which are also continuous distributions of vorticity.

A number of simplifying assumptions are made in the process. Potential-flow (inviscid) methods are used and flow-separation from both leading and trailing edges is modelled by enforcing the Kutta-Joukowksi condition at these points of wake-inception. Further, the flow is assumed to be irrotational (except at solid boundaries and discontinuities in the wake). Although viscosity is generally ignored, its effects are included indirectly in the form of the Kutta-Joukowksi condition and in the formation and shedding of vortices. The wing is taken to be a rigid, flat plate and three-dimensional effects, due to tip vortices or interactions between adjacent wing sections, are ignored. Finally, on the basis of the linearity of the underlying Laplace's equation, the superposition principle is used to calculate the combined effect of the attached and separated flows.

The flow is solved by satisfying the kinematic boundary conditions at the wing surface, the Kutta-Joukowski conditions at the leading and trailing edges and by requiring that the total circulation in a control volume enclosing the system is conserved (Kelvin's law). Conformal transformation is used and all calculations are performed in the circle plane.

As a result, the problem is divided into two distinct components: a wake-free (quasisteady) element and a wake-induced (unsteady) one. By giving the wing six degrees of freedom in terms of displacements and rates of displacement of sweep, heave and pitch, a rather complex kinematic boundary condition is derived. This is then used to derive an expression for the quasi-steady circulation around each wing section.

The effect of the two wakes is then incorporated by computing the additional vorticity 
required to reestablish the boundary conditions described above. What finally results are two coupled, nonlinear, wake integral equations. The first is a generalised and nonlinear form of the original Wagner wake integral equation (Wagner 1925), incorporating the effects of both a nonlinear trailing-edge wake and a leading-edge vortex. It arises from enforcing the Kutta-Joukowski condition at the trailing-edge and is given by

$$
\Gamma_{0}(t)=-\left[\oint_{\text {wake }} \Re\left(\frac{\mathcal{Z}_{\text {wake }}+R}{\mathcal{Z}_{\text {wake }}-R}\right) \gamma_{\text {wake }} \mathrm{d} \mathcal{Z}_{\text {wake }}+\oint_{\text {lev }} \Re\left(\frac{\mathcal{Z}_{\text {lev }}+R}{\mathcal{Z}_{\text {lev }}-R}\right) \gamma_{\text {lev }} \mathrm{d} \mathcal{Z}_{\text {lev }}\right]
$$

where $\Gamma_{0}$ is quasi-steady bound circulation, $\gamma$ is shed vorticity, $\mathcal{Z}$ refers to the coordinates of the shed wakes (in the circle plane), $R$ is the radius of the circle representing the aerofoil, $\Re$

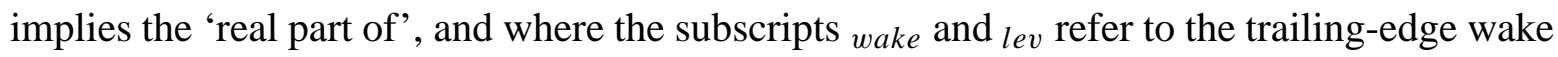
and leading-edge vortex, respectively. As is evident from Equation 19 above, the complex variable is used extensively throughout. The second equation is also similar to the one above but results from requiring stagnation at the leading edge. These two novel equations form the basis upon which this work stands.

The kernel in Wagner's wake integral ( $K$ in Equation 10 ) is linear owing to the flat shed wake. By contrast, in Equation 19, nonlinearity is expressed not only by the form of the kernel but also due to the nonlinear shed wake. The nonlinearity is further reinforced by the presence of two wake integrals instead of one (cf. Equations 10 and 19 .

Forces are computed by Kelvin's method of impulses (Thomson 1910, von Kármán $\&$ Sears 1938, Wu 1981). The bound and shed vortices constitute vortex pairs that impart impulses between them. The combined time-rate-of-change of impulse of all vortex pairs is a measure of the force on the wing (since only the bound vortices sustain Kutta-Joukowski forces). Moment is computed similarly from the time-rate-of-change of moment of impulse. For hover, the aerodynamic moment about the wing pitching axis (described in the physical plane) is given by

$$
M=\frac{\mathrm{d}}{\mathrm{d} t}\left(\frac{\rho}{2} \oint|\zeta|^{2} \gamma \mathrm{d} \zeta\right)-\Im\left(\overline{U_{0}} \cdot \imath \rho \oint \zeta \gamma \mathrm{d} \zeta\right)
$$

where $\rho$ is fluid density, $\zeta$ is the coordinate of a generic (bound, trailing-edge or leading-edge) vortex relative to the wing pitch axis, $\gamma$ is the vorticity associated with the generic vortex, $\overline{U_{0}}$ is the complex conjugate of the complex velocity of the wing pitch axis relative to an inertial coordinate system, and $\Im$ implies the 'imaginary part of'. Comparison of Equation 20 with Equation 10 again impresses the nonlinearity captured by the current unsteady aerodynamic model.

\subsection{Computational implementation}

The governing equations developed above are exact (within the limits of the assumptions made) but nonlinear and, as such, do not have a closed-form solution. Solutions are, therefore, found numerically using the discrete vortex method (Ansari et al. 2006b) whereby point vortices are used as the Lagrangian markers for the integrals (for full details, see Ansari 2004). Motion begins from rest when there is no wake, i.e. total circulation is zero. The approach is 
formulated as an initial-value problem using these initial conditions and the flow is solved for all subsequent times using a time-marching algorithm.

The aerofoil is represented by a distribution of discrete point vortices, as are the leadingedge vortex and the trailing-edge wake. At each time-step, the quasi-steady bound circulation is computed for smooth flow at the trailing edge. Two new vortices are then released, one each from the leading and trailing edges, and placed such that they follow the trace left by the previous shed vortex. The above nonlinear equations are then solved simultaneously for the circulation strengths of the two new vortices.

At the end of the time-step, the solution is marched forward in time by convecting the shed vortices in the wake using a forward Euler scheme. Convection is governed by a discrete form of the Rott-Birkhoff equation (Rott 1956, Birkhoff 1962). These vortices move with the local fluid velocity because they are 'free', and hence, cannot sustain Kutta-Joukowski forces. During the more acute phases of the flapping cycle (e.g. stroke reversals), the time-steps are subdivided into finer sub-time-steps to give better resolution but at the cost of increased CPU flops. A spin-off of this method is that flow-visualisation is automatically generated (see Figure 3).

\subsection{Results from aerodynamic model}

The validity of the aerodynamic model was established by comparing with existing experimental data. Comparisons were, therefore, drawn with both flow-visualisation and force data. Flowfield comparison was made with the experiments of Dickinson \& Götz (1993) where a wing at high angle of attack was impulsively started and subsequently moved with constant speed at $R e \approx 200$, and good agreement was found (see Figure 3).

Comparison of force data was made with a later set of experiments by Dickinson (priv. comm.) on his Robofly - a mechanical, scaled-up fruit-fly wing executing insect-like kinematics in a tank of mineral oil at $R e \approx 160$ (see also Birch \& Dickinson 2003). Both lift and drag predictions were found again to be consistent with experimental data (see Figure 6). In addition to showing the unsteadiness of the flow, the figure also demonstrates the periodic nature of the forces generated. This observation is of particular relevance to the discussion of oscillatory solutions earlier $(\$ 3.3 .1)$.

\subsection{Relevance to flight dynamics}

The aerodynamic model of insect-like hover presented here provides theoretical means of producing, from first principles, a representation of the aerodynamic moment, see (20), as in the longitudinal pitching moment example considered on page 10. Integral equation (10) considered there was linear and could be approximated with 11] with both coefficients $M_{\alpha}$ and $M_{\dot{\alpha}}$ constant. By contrast, the aerodynamic model described here results in a nonlinear integral equation, see 20), and thus its approximation suitable for flight dynamic purposes should follow (13), i.e. with $M_{\alpha}$ and $M_{\dot{\alpha}}$ in (11) depending on $\alpha$ and $\dot{\alpha}$.

In practice, the force and moment equations defining the aerodynamic model for hover

are solved numerically, see Section 4.2 . For a given time history of body kinematics, the 


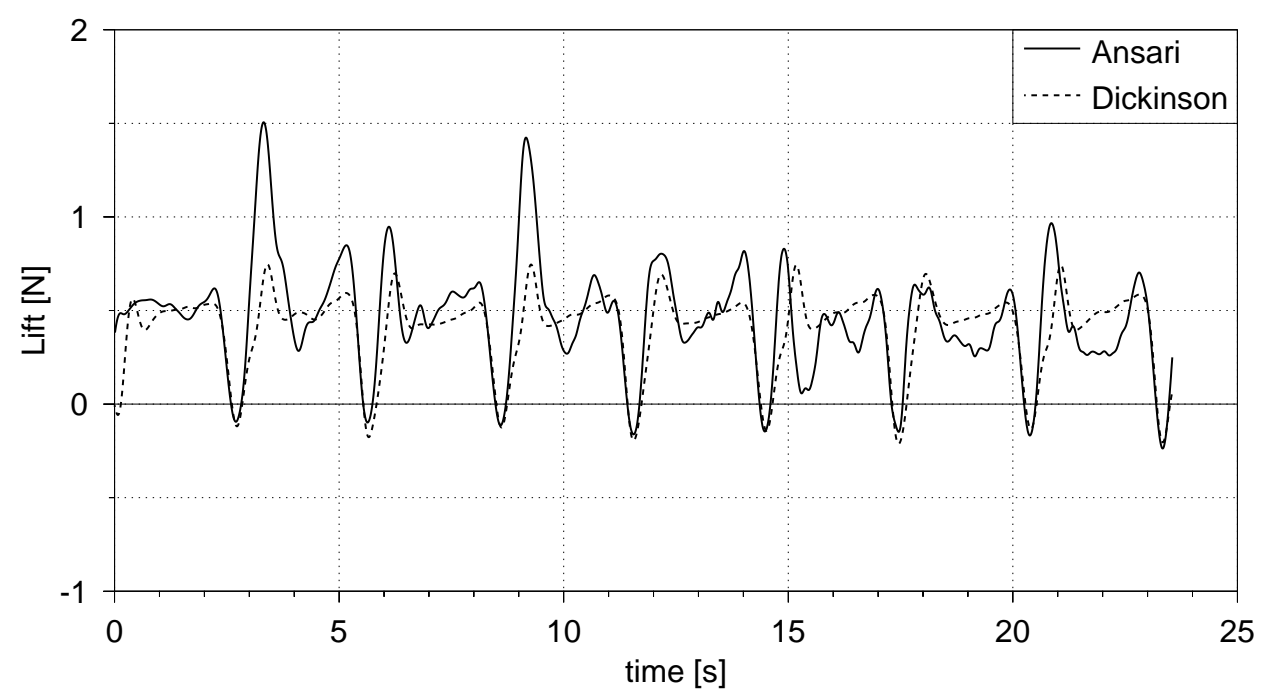

Figure 6. Comparison between lift prediction from aerodynamic model (Ansari 2004) and that obtained through experiment (Dickinson priv. comm.)

corresponding time history of $\boldsymbol{F}(t)$ and $\boldsymbol{M}(t)$ is calculated. From the calculated results one can tabulate the values of $\boldsymbol{M}(t)$ against the values of $\alpha(t)$ and $\dot{\alpha}(t)$ and obtain (through interpolation) a relationship of the type (13). Of course, in the case of hover such relationships are trivial, as the body kinematics is zero. However, for fundamental manoeuvres, see page 9. and/or mild transitions between fundamental manoeuvres, the relationships will be nontrivial, as the body kinematics will be non-zero. Then an appropriate aerodynamic model (extended from hover) will yield the required relationships from first principles. This way, the aerodynamic data required for flight dynamics equations of motion will be generated theoretically, without recourse to challenging experiments.

\section{Conclusions}

Flight dynamics of insect flight can be described by the standard six-degrees-of-freedom equations of motion in which the components of the aerodynamic force and moment are periodic. The key issue is obtaining relationships between these components and the body kinematics for the flight regimes of interest. In conventional aeronautics this usually is done empirically, but in the context of insects the experimental difficulties are much greater. This is not only due to the smallness and fragility of insects, but—above all—due to the difficulties of accounting for active flight control applied by the insect during the experiment. Therefore, an attractive alternative is to consider ways of producing the required aerodynamic data from first principles either by aerodynamic modelling or through computational fluid dynamics (CFD).

Aerodynamic modelling progresses from first principles, but introduces several simplifications to the basic equations of fluid mechanics, justified by the known flow phenomenology and/or geometric and kinematic symmetries. Such justifiable simplifications are present in special flight regimes, most notably hover, and distinguish these regimes by tractability of the associated aerodynamics. This led us to define six types of fundamental 
manoeuvres, i.e. those steady flight conditions for which only one component of both the translational and rotational body velocities is non-zero (and constant). We believe that for these flight regimes it is feasible to derive aerodynamic models by extension of the model for hover briefly presented here. For more complicated flight regimes, a recourse to computational fluid dynamics (CFD) will be needed in order theoretically to generate the aerodynamic data required for the flight dynamics equations of motion.

Since the aerodynamic force and moment production in insects is periodic, it is relevant to consider specialised mathematical tools which deal with nonlinear differential equations forced by nonlinear oscillations. In particular, the existence (or otherwise) of periodic solutions has direct meaning in the flight dynamics context. Indeed, if all solutions of the equations of motion were periodic, straight line flight (observed in insects) would not be possible. On the other hand, butterflies often fly "erratically" which may mean that they employ quasi-periodic (or even chaotic) solutions in order to avoid capture by predators.

Computational generation of the aerodynamic data for the purposes of flight dynamics equations, and also deeper analysis of periodic solutions of the equations, require further progress in aerodynamic or CFD modelling of insect flapping. It should be emphasised that while this calls largely for theoretical progress, the resulting fluid dynamic predictions must be verified by flow visualisation and measurement. Even proceeding from first principles of fluid mechanics must be accompanied by empirical verifications if only to check that the mathematical assumptions made indeed reflect the physical realities of the flow.

\section{Acknowledgements}

This work was supported by the AFOSR Contract No. F61775-02-C4048 and EPSRC Grant GR/M78472/01.

\section{References}

Allgower, E. L. \& Georg, K. (1990), Numerical Continuation Methods. An Introduction, Springer, Berlin.

Ansari, S. A. (2004), A Nonlinear, Unsteady, Aerodynamic Model for Insect-like Flapping Wings in the Hover with Micro Air Vehicle Applications, PhD thesis, Cranfield University (RMCS Shrivenham).

Ansari, S. A., Knowles, K. \& Żbikowski, R. (2003), Aerodynamic Modelling of Some Planforms for Insectlike Flapping Wings, in 'The Royal Aeronautical Society CEAS Aerospace Aerodynamics Conference', London, pp. 38.1-38.14. 10-12 June.

Ansari, S. A., Żbikowski, R. \& Knowles, K. (2006a), A nonlinear unsteady aerodynamic model for insect-like flapping wings in the hover: Part I. Methodology and analysis. Accepted for publication in IMechE Journal of Aerospace Engineering: Part G.

Ansari, S. A., Żbikowski, R. \& Knowles, K. (2006b), A nonlinear unsteady aerodynamic model for insect-like flapping wings in the hover: Part II. Implementation and validation. Accepted for publication in IMechE Journal of Aerospace Engineering: Part G.

Arnol'd, V. I., Afrajmovich, V. S., Il'yashenko, Y. S. \& Shil'nikov, L. P. (1999), Bifurcation Theory and Catastrophe Theory, Springer, Berlin.

Avanzini, G. \& de Matteis, G. (1997), 'Bifurcation analysis of a highly augmented aircraft model', Journal of Guidance, Control, and Dynamics 20(4), 754-759. 
Birch, J. M. \& Dickinson, M. H. (2001), 'Spanwise Flow and the Attachment of the Leading-Edge Vortex on Insect Wings', Nature 412(6848), 729-733.

Birch, J. M. \& Dickinson, M. H. (2003), 'The influence of wing-wake interactions on the production of aerodynamic forces in flapping flight', Journal of Experimental Biology 206, 2257-2272.

Birkhoff, G. (1962), Helmholtz and Taylor Instability, in 'Proceedings of the Symposium on Applied Mathematics', Vol. 13, American Mathematical Society, pp. 55-76.

Carroll, J. V. \& Mehra, R. K. (1982), 'Bifurcation analysis of nonlinear aircraft dynamics', Journal of Guidance, Control, and Dynamics 5(5), 529-536.

Chow, S.-N. \& Hale, J. K. (1982), Methods of Bifurcation Theory, Springer, New York.

Demazure, M. (2000), Bifurcations and Catastrophes. Geometry of Solutions to Nonlinear Problems, Springer, Berlin.

Dickinson, M. H. (1994), 'The Effects of Wing Rotation on Unsteady Aerodynamic Performance at Low Reynolds Numbers', Journal of Experimental Biology 192, 179-206.

Dickinson, M. H. (priv. comm.), '2003 California Institute of Technology, Pasadena, CA'.

Dickinson, M. H. \& Götz, K. G. (1993), 'Unsteady Aerodynamic Performance of Model Wings at Low Reynolds Numbers', Journal of Experimental Biology 174, 45-64.

Dickinson, M. H., Lehmann, F.-O. \& Sane, S. P. (1999), 'Wing Rotation and the Aerodynamic Basis of Insect Flight', Science 284, 1954-1960.

Dudley, R. \& Ellington, C. P. (1990), 'Mechanics of Forward Flight in Bumblebees. II. Quasi-Steady Lift and Power Requirements', Journal of Experimental Biology 148, 53-88.

Ellington, C. P. (1984), 'The Aerodynamics of Hovering Insect Flight: III. Kinematics', Philosophical Transactions of the Royal Society of London Series B 305, 41-78.

Ellington, C. P., van den Berg, C., Willmott, A. P. \& Thomas, A. L. R. (1996), 'Leading-edge vortices in insect flight', Nature 384, 626-630.

Ennos, A. R. (1989a), 'Inertial and Aerodynamic Torques on the Wings of Diptera in Flight', Journal of Experimental Biology 142, 87-95.

Ennos, A. R. (1989b), 'The Kinematics and Aerodynamics of the Free Flight of Some Diptera', Journal of Experimental Biology 142, 49-85.

Etkin, B. \& Reid, L. D. (1996), Dynamics of Flight. Stability and Control, Third edn, Wiley, New York.

Fradkov, A. L. \& Pogromsky, A. Y. (1998), Introduction to control of oscillations and chaos, World Scinetific, Singapore.

Galiński, C. \& Żbikowski, R. (2005), 'Insect-like flapping wing mechanism based on a double spherical Scotch yoke', Journal of the Royal Society Interface 2(3), 223-235.

Goman, M. G., Zagainov, G. I. \& Khramtsovsky, A. V. (1997), 'Application of bifurcation methods to nonlinear flight dynamics problems', Progress in Aerospace Sciences 33(9-10), 539-586.

Govaerts, W. J. F. (2000), Numerical Methods for Bifurcations of Dynamical Equlibria, SIAM, Philadelphia.

Gránásy, P. \& Thomasson, P. G. (1998), 'Non-linear flight dynamics at high angles-of-attack', Aeronautical Journal 102(1016), 337-343.

Grodnitsky, D. L. \& Morozov, P. P. (1993), 'Vortex Formation During Tethered Flight of Functionally and Morphologically Two-Winged Insects, Including Evolutionary Considerations on Insect Flight', Journal of Experimental Biology 182, 11-40.

Hancock, G. J. (1995), An Introduction to the Flight Dynamics of Rigid Aeroplanes, Ellis Horwood, New York.

Huijberts, H. J. C. \& Nijmeijer, H. (2001), An observer view on synchronization, in A. Isidori, F. LamnabhiLagarrigue \& W. Respondek, eds, 'Nonlinear Control in the Year 2000', Vol. 1, Springer-Verlag.

Jahnke, C. C. \& Culick, F. E. C. (1994), 'Application of bifurcation theory to the high-angle-of-attack dynamics of the F-14', Journal of Aircraft 31(1), 26-34.

Kato, J., Martynyuk, A. A. \& Shestakov, A. A. (1996), Stability of Motion of Nonautonomous Systems (Method of Limiting Equations), Gordon and Breach Publishers, Luxembourg.

LaSalle, J. P. (1976), The Stability of Dynamical Systems, SIAM, Philadelphia, PA. (With Appendix "Limiting Equations and Stability of Nonautonomous Ordinary Differential Equations" by Z. Artstein).

Liaw, D.-C. \& Song, C.-C. (2001), 'Analysis of longitudinal flight dynamics: A bifurcation-theoretic approach', 
Journal of Guidance, Control, and Dynamics 24(1), 109-116.

Liaw, D.-C., Song, C.-C., Liang, Y.-W. \& Chung, W.-C. (2003), 'Two-parameter bifurcation analysis of longitudinal flight dynamics', IEEE Transactions on Aerospace and Electronic Systems 39(3), 11031112.

Liu, H., Ellington, C. P., Kawachi, K., van den Berg, C. \& Wilmott, A. P. (1998), 'A Computational Fluid Dynamic Study of Hawkmoth Hovering', Journal of Experimental Biology 201, 461-477.

Lowenberg, M. H. \& Champneys, A. R. (1998), 'Shil'nikov homoclinic dynamics and the escape from roll autorotation in an F-4 model', Philosophical Transactions of the Royal Society of London (Series A: Mathematical, Physical and Engineering Sciences) 356(1745), 2241-2256.

Lowenberg, M. H. \& Patel, Y. (2000), 'Use of bifurcation diagrams in piloted test procedures', Aeronautical Journal 104(1035), 225-235.

Markus, L. (1956), Asymptotically autonomous differential equations, in S. Lefschetz, ed., 'Contributions to the theory of nonlinear oscillations', Vol. 3, Princeton University Press, pp. 17-29.

Martin, L. J. \& Carpenter, P. W. (1977), 'Flow-visualisation experiments on butterflies in simulated gliding flight', Fortschritte der Zoologie 24(2/3), 307-316.

McCune, J. E., Lam, C.-M. G. \& Scott, M. T. (1990), 'Nonlinear Aerodynamics of Two-Dimensional Airfoils in Severe Maneuver', AIAA Journal 28(3), 385-393.

McCune, J. E. \& Tavares, T. S. (1993), 'Perspective: Unsteady wing theory - the Kármán/Sears legacy', Transactions of the ASME: Journal of Fluids Engineering 115(4), 548-560.

Orlik-Rückemann, K. J. (1983), 'Aerodynamic aspects of aircraft dynamics at high angles of attack', Journal of Aircraft 20(9), 737-752.

Patel, Y. \& Littleboy, D. (1998), 'Piloted simulation tools for aircraft departure analysis', Philosophical Transactions of the Royal Society of London (Series A: Mathematical, Physical and Engineering Sciences) 356(1745), 2203-2221.

Pedersen, C. B. (2003), An indicial-Polhamus model of aerodynamics of insect-like flapping wings in hover, PhD thesis, Cranfield University (RMCS Shrivenham).

Pikovsky, A., Rosenblum, M. \& Kurths, J. (2001), Sunchronization. A universal concept in onlinear sciences, Cambridge University Press, Cambridge, England.

Roskam, J. (1995), Airplane Flight Dynamics and Automatic Flight Controls. Part I, DARcorporation, Lawrence, KS.

Rott, N. (1956), 'Diffraction of a weak shock with vortex generation', Journal of Fluid Mechanics pp. 111-128.

Sane, S. P. \& Dickinson, M. H. (2001), 'The Control and Flight Force by a Flapping Wing: Lift and Drag Production', Journal of Experimental Biology 204, 2607-2626.

Sauer, T., Yorke, J. A. \& Casdagli, M. (1991), 'Embedology', Journal of Statistical Physics 65(3/4), 579-616.

Sinha, N. K. (2002), 'Application of bifurcation methods to F-18/HARV open-loop dynamics in landing configurations', Defence Science Journal 52(2), 103-115.

Strauss, A. \& Yorke, J. A. (1967), 'On asymptotically autonomous differential equations', Mathematical Systems Theory $1(2), 175-182$.

Taylor, G. K. \& Thomas, A. L. R. (2003), 'Dynamic flight stability in the desert locust Schistocerca gregaria', Journal of Experimental Biology 206(16), 2803-2829.

Taylor, G. K. \& Żbikowski, R. (2005), 'Nonlinear time-periodic models of the longitudinal flight dynamics of desert locusts Schistocerca gregaria', Journal of the Royal Society Interface 2(3), 197-221.

Thomas, H. H. B. M. (1984), 'Some thoughts on mathematical models for flight dynamics', Aeronautical Journal 88(875), 169-178.

Thomson, W. (1910), On Vortex Motion, in 'Mathematical and Physical Papers: Volume IV Hydrodynamics and General Dynamics', Cambridge University Press. a.k.a. Lord Kelvin.

Tobak, M., Chapman, G. T. \& Schiff, L. B. (1984), Mathematical modeling of the aerodynamic characteristics in flight dynamics, NASA Technical Memorandum 85880, National Aeronautics and Space Administration.

Tobak, M. \& Schiff, L. B. (1978), The role of time-history effects in the formulation of the aerodynamics of aircraft dynamics, in 'Dynamic Stability Parameters', Vol. 235 of AGARD Conference Proceedings, 
pp. 26.1-26.10.

van den Berg, C. \& Ellington, C. P. (1997), 'The Three-Dimensional Leading-Edge Vortex of a "Hovering" Model Hawkmoth', Philosophical Transactions of the Royal Society of London Series B 352(1351), 329340.

von Kármán, T. \& Sears, W. R. (1938), ‘Airfoil Theory for Non-Uniform Motion’, Journal of the Aeronautical Sciences 5(10), 379-390.

Wagner, H. (1925), 'Über die Entstehung des dynamischen Auftriebes von Tragflügeln', Zeitschrift für Angewandte Mathematik und Mechanik 5(1), 17-35.

Wakeling, J. M. \& Ellington, C. P. (1997), 'Dragonfly Flight: III. Lift and Power Requirements', Journal of Experimental Biology 200, 583-600.

Willmott, A. P., Ellington, C. P. \& Thomas, A. L. R. (1997), 'Flow Visualization and Unsteady Aerodynamics in the Flight of the Hawkmoth, Manduca sexta', Philosophical Transactions of the Royal Society of London Series B 352, 303-316.

Wu, J. C. (1981), 'Theory for Aerodynamic Force and Moment in Viscous Flow', AIAA Journal 19(4), $434-441$.

Wu, Y. (1995), 'Oscillation via limiting equations', Nonlinear Analysis, Theory, Methods \& Applications 25(1), 89-102.

Yakubovich, V. A. \& Starzhinskii, V. M. (1975), Linear Differential Equations with Periodic Coefficients, Vol. 1, Wiley, New York.

Żbikowski, R. (1999a), Flapping Wing Autonomous Micro Air Vehicles: Research Programme Outline, in 'Fourteenth International Conference on Unmanned Air Vehicle Systems', Vol. Supplementary Papers, pp. 38.1-38.5.

Żbikowski, R. (1999b), Flapping Wing Micro Air Vehicle: A Guided Platform for Microsensors, in 'Royal Aeronautical Society Conference on Nanotechnology and Microengineering for Future Guided Weapons', pp. 1.1-1.11.

Żbikowski, R. (2002), 'On aerodynamic modelling of an insect-like flapping wing in hover for micro air vehicles', Philosophical Transactions of the Royal Society of London (Series A: Mathematical, Physical and Engineering Sciences) 360(1791), 273-290.

Żbikowski, R. (2004), 'Sensor rich feedback control', IEEE Instrumentation \& Measurement Magazine 7(3), 1926.

Żbikowski, R., Galiński, C. \& Pedersen, C. B. (2005), 'A four-bar linkage mechanism for insect-like flapping wings in hover: Concept and an outline of its realisation', Journal of Mechanical Design 127(4), 817824.

Żbikowski, R., Knowles, K., Pedersen, C. B. \& Galiński, C. (2004), 'Some aeromechanical aspects of insectlike flapping wings in hover', Proceedings of the Institution of Mechanical Engineers Part G: Journal of Aerospace Eengineering 218(G6), 389-398. 
2006-07-10

On mathematical modelling of insect flight dynamics in the context of micro air vehicles

\section{Zbikowski, Rafal \\ IOP Publishing}

Rafal Zbikowski, Salman A. Ansari and Kevin Knowles. On mathematical modelling of insect flight dynamics in the context of micro air vehicles. Bioinspiration \& Biomimetics, Volume 1, Number 2, ppR-26-R37

http://dx.doi.org/10.1088/1748-3182/1/2/R02

Downloaded from Cranfield Library Services E-Repository 